\title{
Un Art de réussir à la cour de Rome: L'Idea del Prelato de Baldovino del Monte
}

\author{
PIERRE HURTUBISE
}

L'existence, d'une part, de la monumentale Histoire des Papes de Ludwig von Pastor, ${ }^{1}$ de l'autre, de toute une série d'études du type de celles que Rodocanachi produisit au début de ce siècle ${ }^{2}$ a créé pendant longtemps l'illusion que nous savions tout ou à peu près tout ce qu'il était possible de savoir sur la cour des papes au XVI ${ }^{e}$ siècle. J'étais moi-même de cette persuasion jusqu'a ce que, il y a trois ans, je me mette sérieusement à l'étude de cette cour. Ma conviction maintenant est que la recherche sur le sujet est à peine amorcée et qu'il faudrait plutôt parler, comme le faisait récemment Paolo Prodi dans son ouvrage sur la papauté aux XVI et $\mathrm{XVII}^{\mathrm{e}}$ siècles, d'un domaine totalement négligé de recherche. ${ }^{3}$

La présente étude se situe dans le cadre d'une enquête de longue haleine visant justement à mettre fin à cette négligence. Elle vise à répondre à une des nombreuses questions qu'inévitablement on se pose au fur et à mesure que l'on avance dans l'étude de ce dossier, question d'ailleurs que les contemporains eux-mêmes se posaient à l'époque, à savoir: comment entrait-on à la cour et, une fois entré, comment pouvait-on espérer y réussir?

J'ai présentement en filière les noms de 4 ou 5000 courtisans (et curialistes) ayant fait partie de la cour pontificale au $\mathrm{XVI}^{\mathrm{e}}$ siècle et pourrai, éventuellement, je l'espère, avec l'aide de l'ordinateur, retracer les carrières de bon nombre d'entre eux. Restera alors le problème d'expliquer les réussites des uns, les échecs ou, du moins, les moindres réussites des autres. Ces réussites (ou ces échecs) s'expliquaient-ils par la seule bonne (ou mauvaise) fortune, par le seul concours d'heureuses (ou funestes) circonstances? Pouvait-on être l'artisan de son propre succès et, si oui, existait-il des recettes sûres et éprouvées, susceptibles de conduire à un tel succès? Il ne manquait pas au XVI ${ }^{e}$ siècle d'individus pour le croire et ces derniers produiront toute une série de traités en ce sens, traités que je suggérerais de regrouper sous le titre d'Arts de réussir à la cour, la cour étant, bien entendu, dans le cas qui nous occupe ici, celle de Rome. ${ }^{4}$ L'idea del Prelato de Baldovino del Monte dont il sera ici principalement question est un des meilleurs exemples 
du genre et il a cette particularité d'avoir été publié à l'époque, ce qui, à ma connaissance, n'est pas le cas des autres. ${ }^{5}$

$$
* * *
$$

Un mot tout d'abord du texte lui-même et de son auteur. S'il faut en croire l'éditeur de L'idea del Prelato, un certain Giovanni Pieroni qui se dit d'ailleurs protégé de Baldovino del Monte, ce dernier aurait écrit son ouvrage pour un ami en partance pour la cour de Rome. Mais le texte n'était pas destiné à la publication et c'est Pieroni qui aurait pris l'initiative de le faire imprimer, convaincu, disait-il, qu'un ouvrage aussi remarquable et utile sedevait d'être connu d'un plus large public. D'où sa décision de dédier $L$ "idea del Prelato à Fabrizio Coloreto, chambellan du grand-duc de Toscane qui, il l'espérait, lui obtiendrait l'indulgence et la compréhension de l'auteur. ${ }^{6}$

Il faudrait être quelque peu naîf pour prendre à la lettre ces "précautions" de Pieroni qui visaient probablement plus à mettre en appétit des lecteurs éventuels qu'à apaiser le courroux d'un auteur publié à son insu. Il est plus que vraisemblable que ce dernier savait très bien ce qui se passait et qu'il en était d'ailleurs fort aise. Mais l'éditeur ajoute à notre confusion, probablement encore ici de connivence avec Baldovino del Monte, en faisant de ce dernier l'auteur de L'idea del Prelato, car, lorsque nous lisons la préface écrite par ledit Baldovino, préface qui suit immédiatement la présentation de Pieroni, nous découvrons que le véritable auteur de L'idea del Prelato n'est pas Baldovino del Monte, mais, comme ce dernier nous en assure lui-même avec une fierté évidente, un de ses antenati, le célèbre cardinal Antonio del Monte. Apparement Baldovino s'était limité à transcrire le texte en question, texte qu'il aurait trouvé dans les archives familiales, et à en faire bénéficier un ami dont il venait d'apprendre le départ prochain pour Rome. 'Il ne méritait donc pas le nom d'auteur, mais le seul fait qu'il ait découvert le texte en question, qu'il lui ait en quelque sorte redonné vie suffisait sans doute, du moins aux yeux d'un Giovanni Pieroni, pour que son nom apparût sur la page-titre d'un livre qui, sans lui, n'aurait peut-être jamais vu le jour. Et puis, après tout, n'était-il pas, lui aussi, un illustre réprésentant de la famille del Monte?

A noter que l'année même où parut à Florence L'idea del Prelato, soit en 1616, un autre ouvrage attribué également à Baldovino del Monte fut publié pas le même éditeur sous le titre: Il Simoncello, ovvero della caccia. ${ }^{8}$ Ce second traité semblait de fait convenir beaucoup mieux au personnage - Baldovino del Monte n'était-il pas à l'époque gentilhomme de la Chambre du grand-duc de Toscane? - tout comme un troisième et dernier traité d'ailleurs qu'il fera paraître à Mantoue en 1625 sous le titre Il Cesarino, ovvero Dell'Arte di cavalcare. ${ }^{9}$ 
Mais il n'en était pas là à ses premières armes, car, s'il faut en croire G.B. Febei, auteur des Notizie di scrittori orvietani, rédigées vers 1751, une Laudatio in funere Francisci Medicei parue à Florence en 1614 serait de sa plume de même qu'un Il Vinto, ovvero Consolazione Filosofica in morte di Francesco Principe di Toscana écrit probablement à la même époque. ${ }^{10}$ Chose certaine, c'est bien lui qui prononça, puis fit publier en 1624 à Bologne un De laudibus Virginii Caesarini, hommage bien senti, mais sans doute aussi intéressé à un homme qui laissait une réputation enviable de poète et de savant - Baldovino del Monte n'hésitait pas à le comparer à Pic de la Mirandole - mais dont on ne pouvait surtout oublier qu'il avait été majordome du pape Urbain VIII. C'est d'ailleurs à ce dernier et non à la famille du défunt que del Monte prenait soin de dédier son panégyrique. ${ }^{11}$

Cette seule énumération d'oeuvres effectivement sorties de la plume d'un homme non seulement cultivé, mais connaissant on nepeut mieux le monde de la cour - celle de Florence, bien entendu, mais également, d'après Giovan Vittorio de' Rossi, celles de Mantoue et de Rome ${ }^{12}$ - oblige à se demander si Baldovino del Monte, à son tour, ne nous induit pas en erreur en prétendant n'être que le "découvreur" d'un texte écrit bien avant lui et qu'il n'aurait fait en somme que sortir de l'oubli. Notre hésitation est d'autant plus justifiée que certaines ressemblances de style et de vocabulaire existent entre L'idea del Prelato et les oeuvres de Baldovino del Monte, telles Il Simoncello ou encore Il Cesarino par exemple. On trouve, en particulier, dans les trois ouvrages en question, la même propension à citer en abondance les oeuvres de l'Antiquité aussi bien grecque que latine nous y reviendrons plus loin - et à tirer de ces mêmes sources des exempla servant à illustrer le propos de l'auteur. ${ }^{13}$

Comment, d'autre part, expliquer l'absence de toute référence, voire même de toute allusion au traité du cardinal del Monte dans la diserte littérature de cour du XVIe siècle, quand on sait que ce traité était destiné, de l'aveu même de son auteur, au jeune Giovan Maria del Monte, le futur Jules III? Pareille discrétion surprend surtout dans une ville et à une époque réputées précisément pour le contraire. On sait quelle diffusion connaîtra dans la deuxième moitié du XVI ${ }^{e}$ siècle un traité semblable du cardinal Commendone pourtant lui aussi écrit à l'intention d'une personne bien particulière et non du grand public. ${ }^{14}$

Un examen un peu plus attentif de L'idea del Prelato oblige toutefois à écarter la plupart de ces motifs d'hésitation. Tout d'abord, les ressemblances de style et de vocabulaire évoquées plus haut sont de nature plutôt superficielle et pourraient fort bien n'être que fortuites. Ou encore résulter de corrections, d'améliorations apportées au texte original par Baldovino del Monte au moment de la transcription dudit texte. De fait, L'idea del Prelato est écrit dans un style en général plus lourd que celui de Baldovino del Monte. D’ailleurs, à bien y réfléchir, il sonne trop clérical, fait trop bonnet 
carré pour être d'une plume autre qu'ecclésiastique. Quant aux emprunts massifs aux auteurs de l'Antiquité grecque et latine, cela n'était pas une particularité du XVII' siècle; bien au contraire, le XVII ${ }^{e}$ siècle ne faisait en cela que se conformer à un modèle qui lui venait de la Renaissance.

Mais l'élément le plus important, celui qui, à mes yeux, milite le plus en faveur de l'assertion de Baldovino del Monte, c'est le fait que, pour ce qui est des auteurs cités, pour ce qui est également des faits relatés, L'idea del Prelato ne contient, à proprement parler, aucun anachronisme, c'est-à-dire rien qui permette de situer ce texte à une époque plus tardive que celle indiquée par Baldovino del Monte. Ce dernier fût-il le véritable auteur du traité en question, il faudrait lui supposer une habileté et des connaissances peu communes, ce qui, en l'occurence, paraît difficile à admettre.

Nous partirons donc ici de l'hypothèse que le cardinal Antonio del Monte (ou, du moins, quelqu'un de son entourage et de son époque) est bel et bien l'auteur de L'idea del Prelato. Si tel est le cas, quand et dans quelles circonstances fut composé ce traité?

Tout semble indiquer qu'il le fut peu après l'élévation d'Antonio del Monte au Sacré Collège, c'est-à-dire probablement au printemps ou à l'été de 1511. Le texte, nous l'avons vu, était destiné à son neveu, Giovan Maria del Monte, le futur Jules III, qui, à l'époque, revenait d'études à Pérouse et à Sienne et s'apprêtait à entrer au service de Jules II. ${ }^{15}$ Il est facile de comprendre, à la lumière de ce qu'avait accompli l'oncle et de ce que réussirait plus tard le neveu, que Baldovino del Monte et Giovanni Pieroni aient été intéressés, un siècle plus tard, à promouvoir un texte qui non seulement servait la cause des del Monte, mais pouvait en même temps être présenté comme une formule quasi assurée de succès et donc trouver d'autant plus facilement preneurs.

$* * *$

La formule d'Antonio del Monte était de fait largement fondée, comme il l'assure lui-même au début de son traité, sur son expérience personnelle. S’il était une chose qu'il avait apprise au fil des années, c'était bien que nul ne pouvait espérer réussir à Rome sans une connaissance approfondie du fonctionnement, mais également du climat de la cour pontificale car, insistait-il, la première dignité de cette cour étant élective, Rome était une scène changeante et imprévisible oủ l'on pouvait tout aussi rapidement perdre que gagner. ${ }^{16} \mathrm{Il}$ fallait donc pour y réussir etre constamment sur ses gardes et surtout savoir y manoeuvrer.

Si vuol dunque di questo larghissimo mare conoscer non solo ottimamente il principal porto, e piu capace, che e la grazia del Sommo Pontefice, e proccurare con vele, e con remi di entrarvi, ma i minori ancora; ove ricovrar tu ti possa per 
qualsivoglia fortunoso accidente: ne solo i porti, che securi sono ad ogni vento: come sarebbe a dire l'amicizie de Cardinali, e degli altri gran prelati; ma, per parlare alla marineresca, le cale ancora, e i ridossi della benevolenza dé favorite cortegiani, e delle persone minori. ${ }^{17}$

Le futur courtisan devait donc savoir en tout temps se ménager des contacts à tous les niveaux de la cour: contacts choisis en fonction de leur influence, de leur expérience, mais également de leur accès aux meilleures sources d'information. Le plus humble des serviteurs pouvait, de ce point de vue, s'avérer à l'occasion plus utile que le plus puissant des cardinaux. Il fallait, au fond, savoir exploiter les bonnes sources aux bons moments. L'expérience acquise dans d'autres cours ne suffisait pas. Rome avait un style et des traditions bien à elle. Il fallait apprendre à les connaaitre et à les bien connaitre. Sans quoi tout espoir de réussite était compromis. ${ }^{18}$

Mais comment faisait-on pour entrer à cette cour et, éventuellement, s'y tailler une place? Les ambitions de son neveu étant, comme les siennes, de nature ecclésiastique, Antonio delMonte était d'avis que la première vertu. à cultiver dans ce cas était la vertu de religion ou, si l'on préfère, la piété. Une piété simple, sincère, mais discrète ${ }^{19}$; adaptée d'autre part au type de vie très active dans lequel le neveu allait bientôt s'engager: "A te conviene [ ... ] lasciando da parte Maddalena, tutto a Marta darti in preda."20

Mais il lui fallait aussi, dès le départ, se fixer un but et pas n'importe quel but. Giovan Maria del Monte étant ce qu'il était, ce but ne pouvait être en effet que le plus élevé possible. Tout au moins le chapeau de cardinal. Autrement, il ne valait même pas la peine d'entreprendre une carrière à la cour. Viser haut comportait d'ailleurs de grands avantages psychologiques:

che dovendoti alcun fin proporre nella Romana corte, grande, magnifico, e sublime lo ti proponga; conciosia cosa che, se di conseguirlo non ti avviene per quantunque opere buone tu faccia, che mezzano almeno /... Inon ti potrà mancare; la dove se mezzano tu lo ti proponessi, forse ad infimo, a vile, e di niun pregio di arrivare, t'incontrerebbe. ${ }^{21}$

S'étant fixé un but et ayant mis, pour ainsi dire, Dieu de son côté, à quels moyens, à quelles règles, à quelles stratégies fallait-il avoir recours pour pouvoir réaliser ses ambitions?

Tout le reste du traité, c'est-à-dire quelque 77 pages sur 95 , est consacré à ce problème ou mieux à cet ensemble de problèmes bien concrets, le facteur déterminant étant, dans chaque cas, l'utilité ou pas, la rentabilité ou pas, par rapport au but fixé, de tel ou tel comportement, de telle ou telle attitude, de telle ou telle pratique qu'on pourrait se voir recommander ou être tenté d'adopter. Un certain cynisme colore toute cette partie du traité, bien que, je le reconnais, il soit beaucoup moins accusé que dans certains 
écrits contemporains du même type. Evidemment, on ne peut s'empêcher de penser à Machiavel, bien que Machiavel n'avait pas encore en 1511 publié son Prince, mais le machiavélisme d'Antonio del Monte a ceci de très particulier et de très différent qu'il attache beaucoup de prix à l'honnêteté et à la vertu et croit, contrairement à Machiavel, qu'elles peuvent être, l'une et l'autre, récompensées.

Réussir n'en est pas moins, aux yeux du cardinal, d'abord et avant tout une question de méthode, de prudence et de patience. ${ }^{22}$ Méthode, parce qu'une carrière supposait des étapes, des étapes à franchir selon un certain ordre, voire même selon un certain rythme. Prudence et patience, parce qu'à trop se presser on risquait souvent des erreurs coûteuses et à dévoiler trop tôt ses talents on s'exposait à des jalousies inutiles, en plus d'indisposer ceux dont on pouvait espérer obtenir des grâces:

è tale il costume degli huomini à cui tocca distribuirle, che vogliono, che loro splendido dono, et assolutamente libero apparisca, non merito di chi che sia, le dignitd e le ricchezze, che danno..$^{23}$

Le futur courtisan devait donc procéder avec beaucoup de soin et surtout sans précipitation. Mais il ne pouvait espérer réussir seul, c'est-à-dire sans le concours des autres. Quels autres?

Revenant à un thème déjà abordé dès le début de son traité, le cardinal entreprenait de répondre à cette question en élaborant sur quelque 35 pages ce que l'on pourrait à juste titre appeler: l'art de se faire des alliés (ou des complices?).

Le futur courtisan avait tout d'abord besoin d'amis. De personnes avec lesquelles il se sentit parfaitement à l'aise et à qui il put en toute liberté se confier. Son choix devait se fixer d'abord et avant tout sur des personnes reconnues pour leur vertu. Mais à Rome, capitale de la duplicité, ce n'était pas là chose facile et, nonobstant toutes les règles reconnues de l'amitié, il fallait y rester très prudent dans les rapports qu'on entretenait même avec ses proches. ${ }^{24}$

Le futur courtisan avait surtout besoin de contacts à la cour. Il fallait pour cela qu'il s'y montre souvent ("le visite, gli incontri, gli accompagnamenti.[...] che [...] si fanno tutto giorno") ${ }^{25}$ et que surtout il s'y pénètre des règles, coutumes et pratiques qui y étaient de rigueur. Mais avec qui fallait-il de préférence se lier, à qui fallait-il vouer son temps et ses talents? La réponse à cette question était cyniquement simple. Toute personne, tout groupe de personnes, en état de nuire aussi bien que d'aider, était digne d'attention. Les puissants venaient, bien entendu, en tête de liste, mais également leurs favoris, car ces derniers pouvaient être de précieux alliés dans la conquête des faveurs et de la protection de leur maîtres. Toutes ces personnes, favoris y compris, devaient être traitées à 
l'égal de patrons dans l'espoir que certaines d'entre elles finissent un jour par jouer le rôle qu'on attendait d'elles. ${ }^{26}$

Con quelli, che tù comprenderai, possenti in corte, e di autorità, di porre in opera fa mestiere com'io ti dicea, quella parte della prudenza, che sagacità vien detta, $e$ con essa tutte le piu chiuse, e piu segrete vie investigando, gli umori loro scoprir piu, che si puote, e scoperti, che tu gli abbia, adempir con essi accortamente ogni offizio, che à loro sia di piacere, ed te d'onesto utile esser possa; tutte le voglie loro incontrando, ne à spesa guardando, ne a disagio, che alle tue forze possibil sia..$^{27}$

La flatterie, admettait le cardinal, était, à l'égard de ces personnes, presque de rigueur. Aussi le futur courtisan ne devait-il pas hésiter à y recourir, mais sans la servilité, le mensonge ou la fraude dont certains à l'époque l'assortissaient. Sans doute la distance n'était-elle pas très grand entre ce que le cardinal appelait la sagacité (sagacità), qu'il approuvait, et la ruse (astutia), qu'il rejetait, mais un courtisan pouvait éviter de glisser de l'une à l'autre en se restreignant à des formes très sélectives de flatterie. Par exemple, vanter les traits positifs ou encore les réalisations d'une personne, même si les premiers n'étaient pas nécessairement caractéristiques ou les secondes à mettre réellement au compte de cette personne. Même chose pour la dissimulation (dissimulazione) qu'il fallait parfois se résigner à pratiquer, bien qu'elle ressemblât un peu trop à la simulation (finzione), laquelle ne convenait d'aucune façon à un homme de son état. Mais il fallait être réaliste. Injustement ou chichement traité par son patron, le courtisan devait apprendre à ne rien laisser paraître de son mécontentement ou de sa frustration. Par contre, il ne devait en aucun cas se taire, encore moins paraître approuver lorsque, en sa présence, on disait du mal de ce même patron. ${ }^{28}$

Il y avait donc un temps pour parler et un temps pour se taire. Dans le cas du futur ou encore du jeune courtisan, ce temps était, s'il faut en croire le cardinal, d'une singulière importance. Il fallait, par exemple, se montrer très discret dans les rapports qu'on entretenait avec les grands de la cour. Eviter par-dessus tout d'étaler le fait qu'on était au mieux avec ces derniers: certains patrons n'appréciaient guère ce genre d'exhibitionnisme. Eviter également toute forme d'indiscrétion ou de bavardage concernant ces mêmes patrons et leur entourage. Une particulière attention devait être, de ce point de vue, accordée aux favoris de tous ces grands personnages, ces derniers considérant habituellement les coups portés à leurs subalternes comme des coups portés a euxmêmes. ${ }^{29}$

Mais il ne suffisait pas au courtisan-en-herbe de disposer d'un bon réseau d'amis et de protecteurs: il lui fallait en plus se faire personnellement un nom à la cour.

Encore ici, Antonio del Monte prêchait la discrétion. L'homme d'esprit 
était apprécié de la cour, mais dans la mesure où il savait ménager son talent. Les commérages devaient être laissés aux femmes dont c'était la spécialité. Plusieurs avaient ruiné leurs carrières à Rome pour s'être adonné à l'un et l'autre de ces dangereux passe-temps. ${ }^{30}$

Fa un tuo pensiero, che non solo ogni Cardinale che a Roma vedrai o praticherai per qualsivolgia modo, possa al Papato arrivare, ma che tutti i Prelati ancora, co' quali tu userai amichevolmente, Cardinali esser possano. ${ }^{31}$

Faire trop étalage de ses talents, surtout pour quelqu'un qui amorçait à peine sa carrière, c'était également s'exposer à de graves dangers. Sans doute fallait-il montrer ce dont on était capable afin d'attirer l'attention de ceux qui étaient à même de favoriser les ambitions qu'on nourrissait, mais il fallait le faire par étapes et sans précipitation "come della moneta i ricchi far sogliono, poco, e solo al bisogno mostrarla." ${ }^{2}$ Plus on progressait dans la carrière, plus on pouvait se mettre de l'avant. En ce domaine comme en tant d'autres, l'important était de savoir ménager ses effets.

Mais, poursuivait le cardinal, là où la discrétion n'était plus de mise, c'est lorsque venait le moment d'être ou de se faire l'hôte de membres de la cour. Compte tenu de la carrière qu'il avait en vue, Giovan Maria del Monte devait viser à se créer dès le départune réputation de prélat libéral et munificent. Il fallait à tout prix éviter à ce niveau toute forme, voire même toute apparence d'avarice. Etre trop prêt de ses sous, c'était le plus sûr moyen de compromettre à tout jamais sa carrière. ${ }^{33}$

E quantunque, ela gola, e la pigrizia, e'l piacer delle donne, possano tra le mura delle camere star nascosi, il puzzo dell'avarizia à guisa del fumo, per ogni picciolo spiraglio esce, e si diffonde. ${ }^{34}$

Sans doute fallait-il, d'autre part, fuire la prodigalité, mais l'accent n'en devait pas moins etre mis sur un style de vie digne de ce qu'on était et de ce qu'on représentait. ${ }^{35}$

Aux qualités du courtisan, il fallait, du moins pour la carrière que le cardinal del Monte envisageait pour son neveu, savoir joindre celles du curialiste. Et, à ce propos, le prélat assurait que rien ne serait plus utile à cette carrière qu'une solide maîtrise du latin, langue que malheureusement ses compatriotes négligeaient de plus en plus, alors que les étrangers continuaient à la pratiquer avec bonheur. ${ }^{36}$ Et puisque Giovan Maria serait très probablement appelé à remplir un jour des missions diplomatiques, pourquoi ne se mettrait-il pas à l'étude des principales langues "barbares": le français, l'espagnol, l'allemand par exemple? ${ }^{37}$ Se tenir au courant de ce qui se passait dans ces pays voisins, des caprices, pensées et ambitions de leurs gouvernements ou princes respectifs était également une necessité. De bonnes sources d'information sur ces diverses matières existaient a 
Rome même. Il fallait savoir les exploiter, mais en s'en tenant toujours aux règles de discrétion plus haut indiquées. ${ }^{38}$

Giovan Maria del Monte devait donc multiplier les efforts en vue d'acquérir les connaissances, les habiletés, l'expérience nécessaires à la réalisation de ses projets d'avenir. La paresse était son pire ennemi ("non ha chiunque vien in Corte maggior nimico, o più capitale"). ${ }^{39}$ Qu'il se tint donc constamment occupé. Les temps libres devaient être consacrés soit à des lectures utiles - le cardinal recommandait en particulier les ouvrages d'histoire ou encore les vies des hommes illustres - soit à des retours réguliers sur les choses faites, dites ou entendues en vue de tirer le meilleur parti possible de l'expérience accumulée au fil des jours. ${ }^{40}$ Le cardinal lui conseillait, d'autre part, de se tenir loin de la poésie qui, disait-il, était un passe-temps à réserver aux gens oisifs ("alle oziose persone"). Il reconnaissait la beauté de cet art, mais c'était, disait-il, un art inutile, donc de peu de profit pour lui. Qui plus est, la poésie telle que pratiquée à Rome était intimement liée à l'amour, et l'amour, en plus de faire perdre beaucoup de temps, pouvait facilement mettre en danger une carrière telle que la sienne. ${ }^{41}$.

Mais le talent, l'expérience, une bonne préparation et de puissants patrons ne suffisaient pas pour réussir. Il fallait en plus posséder ce que le cardinal appelait l'instinct du vainqueur, c'est-àdire le goût et la volonté d'arriver au but. Le succès dépendait très souvent de la capacité qu'on avait (ou qu'on n'avait pas) de saisir l'occasion au moment même où elle se présentait. ${ }^{42} \mathrm{~A}$ cet égard, le cardinal signalait l'importance de la "conjoncture" ("congiontura") qu'il décrivait comme "l'unione stessa del tempo, e dell' occasione e de' mezzi, e d'altre favorevoli circunstanze"43. Machiavel n'aurait pu mieux dire.

Mais cela supposait de la part du courtisan une autre essentielle vertu: la vertu de persévérance. Et c'était là, disait Antonio del Monte, une vertu qu'il connaissait bien, ayant dû lui-même la pratiquer, parfois dans des circonstances des plus difficiles.

Ned 'io, qual oggi io mi sia, d cose fatte mi avvenne à un tratto; ma doppo lo essere stato molti anni non conosciuto, non apprezzato in corte, piaqque ad Innocenzo Ottavo di questo nome, la mia industria adoperare, e quinci la mia degnità accresciutasi un poco, gran tempo anche, senza avanzarsi piu oltre, à molti rimase à dreto; ed' avendola promossa e quasi posta in campo Alessandro Sesto, e Pio Terzo augumentatola, Giulio Secondo oggi regnante al segno à che ella e l'ha condotta. ${ }^{44}$

Le cardinal espérait que son neveu ferait preuve d'une égale patience et persévérance et pourrait ainsi connaître un sort au moins égal au sien. Et sur ce souhait plutôt optimiste, il concluait son traité. 
Dans l'analyse que je viens de faire du texte du cardinal del Monte, je me suis limité à l'essentiel, c'est-à-dire aux principaux avis donnés par le prélat à son neveu et aux arguments dont il entoure, dans la plupart des cas, ces avis. Pour rendre justice à son oeuvre, il faudrait également tenir compte du riche assortiment de dicta et d'exempla dont il émaille son discours. Ces dicta et exempla représentent de fait, quantitativement parlant, près de la moitié du texte de l'ouvrage. Le plus frappant, c'est qu'à une ou deux exceptions près, ils sont tous d'origine païenne. A un certain moment, le cardinal sent le besoin de dire: "e potreti di ci non più de'libri profani, ma dalle sacre lettere ancora addure molti esempi," 45 mais la Bible est de fait pratiquement absente de son oeuvre, l'allusion aux vocations divergentes de Marthe et Marie étant le seul lieu biblique explicite de tout l'ouvrage. Comment expliquer une telle discrétion dans un traité écrit après tout par un homme d'Eglise pour un homme d'Eglise s'apprêtant d'ailleurs à entrer au service de la papauté? Il faudrait pour pouvoir répondre à cette question connaître un peu mieux l'auteur de L'idea del Prelato. Or si nous sommes assez bien renseignés sur sa carrière et sur ses exceptionnels talents de juriste et d'administrateur, nous le sommes beaucoup moins en ce qui concerne ses rapports avec la culture de son temps. Sans doute, comme tant d'autres cardinaux et prélats romains, avait-il été touché par les courants humanistes qui occupaient tant de place dans l'Italie de l'époque, mais jusqu'a quel point pouvait-il être considéré comme un humaniste lui-même, il est difficile de le dire. Chose certaine, il connaissait bien ses auteurs anciens et avait tenu à leur faire une large place dans son ouvrage.

De quels auteurs s'agissait-il? De fait, Antonio del Monte cite quelque 23 auteurs différents dont dix grecs et 13 latins. Dans la première catégorie, on trouve en tête de liste Homère avec quatre mentions, suivi d'Isocrate et de Démosthène avec trois mentions chacun, Aristote, Euripide, Thucydide, Epictète, Plutarque, Hérodote, avec deux, et finalement Hésiode, avec une. Dans la seconde catégorie figurent, tout d'abord, loin devant le reste du peloton, Suétone, avec 31 mentions, puis Cicéron, avec six, Catonl'Ancien, avec trois, Ennius Quintus, Juvénal et Quinte-Curce, avec deux, suivis de Valère Maxime, Salluste, Horace, Sénèque, Virgile et Tite-Live avec une mention chacun. Sans doute faudrait-il ajouter à cette dernière catégorie Cassiodore, avec une mention, de fait le seul auteur chrétien cité par le cardinal. On aura remarqué l'importance accordée aux historiens, en particulier à un Suétone. Si Antonio del Monte en recommandait tant la fréquentation à son neveu, c'est qu'il savait d'expérience quel profit il y avait à lire leurs ouvrages.

Pour ce qui est des dicta et exempla retenus par le cardinal, on ne sera pas surpris, à la lumière de ce que nous venons de dire des auteurs cités, de constater qu'ils concernent dans leur très grande majorité des personnages 
de la Rome antique. Ainsi Jules César, mentionné sept fois, l'empereur Auguste, six, Tibère, cinq, Galba, Néron, Caligula, deux chacun, enfin Claude et Vespasien, une fois. Les non Romains, par ailleurs, incluent Philippe de Macédoine et Alexandre le Grand, avec une mention chacun, les empereurs Cyrus et Darius de Perse, le roi Ramiro d'Espagne, Théodoric, roi des Ostrogoths, eux aussi mentionnés une fois.

La liste est impressionnante et montre bien qu'Antonio del Monte avait une culture qui ne se limitait d'aucune façon au monde de la jurisprudence. Comment le jeune Giovan Maria reçut-il ce compendium de sagesse à l'antique? Nous ne le savons pas. Mais il serait surprenant qu'il n'en ait pas été au moins flatté.

En tint-il compte dans la poursuite de sa carrière? Pas complètement, semble-t-il, car s'il s'intéressa peu aux poètes et à la poésie, il n'évita pas pour autant certains des dangers que son oncle disait naître de la pratique de cet art. ${ }^{46}$ Il n'avait, d'autre part, ni la raffinement nila discrétion souhaitée par le cardinal-les contemporains ne manquent pas de souligner, les uns pour s'en féliciter, les autres pour s'en offusquer, sa grande liberté de parole et de comportement ${ }^{47}$ - mais il était un travailleur acharné, un homme habile et intelligent, et il saura, en particulier, avec le temps se créer, comme le lui avait recommandé son oncle, une réputation de prélat libéral et munificent. L'incomparable villa Giulia est encore là aujourd'hui pour en témoigner. Il était surtout très proche des Farnèse. Et c'est ce qui, à la longue, lui vaudra de gravir un à un les échelons menant jusqu'au trône pontifical..$^{48}$ Les conseils de son oncle n'avaient donc que partiellement servis à assurer sa réussite. Mais, comme Antonio del Monte le disait dans son traité, l'important était d'arriver au but. Tout le reste, après tout, n'était que moyens. Eût-il été encore vivant au moment de l'élection pontificale de 1550 , le cardinal au rait certainement été fier de ce neveu qui, tout compte fait, avait réussi, et réussi audelà de toutes ses espérances.

Université de St. Paul

Notes

1 L. Von Pastor, Geschichte der Päpste .... t. III - XI (Fribourg en Brisgau, 1955-59 [rééd.]).

2 E.P. Rodocanachi, Histoire de Rome: une cour princière au Vatican pendant la Renaissance. Sixte IV. Innocent VIII, Alexandre VI (Paris, 1925). ID., Histoire de Rome: le pontificat de Jules II (Paris 1928). ID., Histoire de Rome: le pontificat de Léon X (Paris 1931). ID., Histoire de Rome: les pontificats d'Adrien VI et de Clément VII (Paris, 1933).

3 P. Prodi, $I$ Sovrano Pontifice (Bologne, 1982), p. 102.

4 Le plus célèbre de ces traités est, sans contredit, celui du cardinal Francesco Commendone, Discorso sopra la Cone di Roma, probablement écrit vers 1564-65. Il connut une très large diffusion aux XVI ${ }^{\bullet}$ et XVII ${ }^{e}$ siècles comme en témoignent les nombreuses copies qui nous restent. A ce propos, Cf. L. Von Pastor, op. cit., VII, 661-662. A noter toutefois que l'ouvrage en question contient un long discours d'un ton très réformiste sur la cour pontificale de l'époque qui fait singulièrement contraste avec ce qui suit sur l'art de réussir à cette mẻme cour. Moins connue, 
mais plus représentative peut-être du cynisme que l'on retrouve à divers degrés dans la plupart de ces traités, la très leste et incisive Instruttione per chi vuol entrar nella Corte Romana (connue aussi sous le titre: Avvertimenti bellissimi..per la Corte di Roma) attribuée au comte de Verrua, ambassadeur du duc de Savoie à Rome, et donc probablement de la fin du XVI' ou du début du XVII' siecle. Au sujet de ce dernier, voir $\mathrm{N}$. Bianchi, Le materie politiche relative all'estero degli Archivi di Stato piemontesi (Bologne-Florence, 1876), p. 296. Ce discours n'a pas connu la diffusion du précédent, mais on en trouve un certain nombre de copies notamment à la Bibliothèque Nationale de Paris (v.g. BN, Mél. Colbert 2, fol. 575-591 rv), à la Bibliothèque du Vatican (F. Chigi N. III 84, fol. 193-206r) et à la Biblioteca Nazionale de Florence (Magl. Cl. XXIV, nos. 63 et 65). Je suggère de distinguer ce genre de traités de ceux que l'on pourrait appeler des Arts de vivre à la cour, tel le Courtisan ( $l$ Cortigiano) de Castiglione par exemple.

5 L'idea del Prelato. Trattato del Signor Baldovino di Monte Simoncelli, De' Signori di Viceno, gentil' uomo della Camera del Serenissimo Gran Duca di Toscana (Florence: Zanobi Pignoni, 1616). [V]95 p. J'ai utilisé un des cinq exemplaires de l'ouvrage existant à la Bibliothèque du Vatican (Cote: BAV R.I. IV 1895 int. 5) et toutes les références qui suivent sont faites à partir de l'exemplaire en question.

6 Ibid., p. [II] - [V].

7 Ibid., P. 1-2.

8 I Simoncello ovvero Della Caccia. Dialogo di Baldovino di Monte Simoncelli (Florence: Zanobi Pignoni, 1616), $131 \mathrm{p}$.

9 Il Cesarino ovvero Dell'Arte di Cavalcare. Dialogo di Baldovino di Monte Simoncelli de' Sig. ri di Viceno nell' Accademia degli Invaghiti detto il Securo (Mantoue, 1625), [XVI] - 151 p.

10 G.B. Febei, "Notizie di scrittori orvietani ..., " dans Archivio Storico per le Marche e per l'Umbria (Foligno, 1886), III, 409-410.

11 De Laudibus Virginii Caesarini, Urbani Octavi P.M. Cubuculi (sic) Praefecti. Oratio Habita Bononiae in Gelatorum Academia (Bologne, 1624), [IV] - 22 p.

12 [G.V. Rossi], Pinacotheca Altera Imaginum Illustrium doctrinae vel ingenii laude, virorum qui auctore superstite, diem suum obierunt (Cologne, 1645), p. 130-136.

13 Il Cesarino, par exemple, cite près d'une cinquantaine d'auteurs anciens, les préférences de l'auteur allant notamment à un Tite-Live, à un Suétone, à un Virgile, à un Jules César, à un Varron, à un Quinte-Curce, à un Xénophon auxquels il emprunte d'abondants dicta et exempla.

14 Le traité de Commendone avait en effet été écrit à l'intention de Girolamo Savorgnano. Cf. L. Von Pastor, op. cit., VII, 662.

15 Ibid., VI, 36-37.

16 L'idea del Prelato, p. 4-5.

17 Ibid., p. 6.

18 Ibid., p. 7-9.

19 Ibid., p. 10-13.

20 Ibid., p. 11.

21 Ibid., p. 13-14.

22 Ibid., p. 18-22.

23 Ibid., p. 19-20.

24 Ibid., p. 22-31.

25 Ibid., p. 31.

26 Ibid., p. 31-34.

27 Ibid., p. 35.

28 Ibid., p. 36-39.

29 Ibid., p. 40-42.

30 Ibid., p. 43-44.

31 Ibid., p. 44. 
32 Ibid., p. 49.

33 lbid., p. 50.

34 Ibid., p. 51.

35 Ibid., p. 52-55.

36 Ibid., p. 57.

37 lbid., p. 58.

38 Ibid., p. 66-68.

39 Ibid., p. 80.

40 Ibid., p. 82-84.

41 lbid., p. 60-63.

42 Ibid., p. 70-71.

43 Ibid., p. 72.

44 Ibid., p. 94.

45 Ibid., p. 81.

46 L. Von Pastor, op. cit., VI, 48 et suiv.

47 Diario di Giulio IIIo, BAV, Urb. lat. 1640, fol. 431 rv.

48 Ibid., fol. $432 v-433 r$. 